\title{
Prospects for Discovering Pulsars in Future Continuum Surveys Using Variance Imaging
}

\author{
S. Dai ${ }^{1 \star}$, S. Johnston ${ }^{1}$, G. Hobbs ${ }^{1}$ \\ ${ }^{1}$ CSIRO Astronomy and Space Science, Australia Telescope National Facility, Box 76 Epping NSW 1710, Australia
}

Accepted XXX. Received YYY; in original form ZZZ

\begin{abstract}
In Dai et al. (2016) we developed a formalism for computing variance images from standard, interferometric radio images containing time and frequency information. Variance imaging with future radio continuum surveys allows us to identify radio pulsars and serves as a complement to conventional pulsar searches which are most sensitive to strictly periodic signals. Here, we carry out simulations to predict the number of pulsars that we can uncover with variance imaging on future continuum surveys. We show that the Australian SKA Pathfinder (ASKAP) Evolutionary Map of the Universe (EMU) survey can find $\sim 30$ normal pulsars and $\sim 40$ millisecond pulsars (MSPs) over and above the number known today, and similarly an all-sky continuum survey with SKA-MID can discover 140 normal pulsars and $\sim 110$ MSPs with this technique. Variance imaging with EMU and SKA-MID will detect pulsars with large duty cycles and is therefore a potential tool for finding MSPs and pulsars in relativistic binary systems. Compared with current pulsar surveys at high Galactic latitudes in the southern hemisphere, variance imaging with EMU and SKA-MID will be more sensitive, and will enable detection of pulsars with dispersion measures between $\sim 10$ and $100 \mathrm{~cm}^{-3} \mathrm{pc}$.
\end{abstract}

Key words: methods: observational - radio continuum: general - pulsars: general

\section{INTRODUCTION}

The next-generation of radio continuum surveys will provide us with ultra-deep images of large swathes of the sky (e.g., Norris et al. 2011; Prandoni \& Seymour 2015). As a result, millions of radio sources will be detected, a tiny (but significant) fraction of which will be radio pulsars. How can we distinguish radio pulsars from all the other sources? In Dai et al. (2016) (hereafter D16), we proposed to use the variance of flux densities caused by diffractive interstellar scintillation to distinguish pulsars from other radio sources. In conjunction with the conventional all-sky blind searches for pulsars, this technique has the potential to uncover pulsars missed by the standard techniques. This is because variance imaging can detect pulsars independent of their pulse width. This is not the case with conventional pulsar searches which are most sensitive to narrow pulse-widths. A variety of effects can broaden the pulse profile, including dispersion-measure (DM) smearing, scattering and orbital modulation of spin periods. This makes it possible to identify sub-millisecond pulsars, pulsar-blackhole systems and pulsars in the Galactic centre in continuum surveys.

^ E-mail: shi.dai@csiro.au
However, variance imaging also has its limitations. In D16, we investigated the properties of variance imaging and showed that the sensitivity peaks for pulsars whose scintillation bandwidth and time-scales are close to the channel bandwidth and subintegration time of a particular continuum image. Therefore, for a given continuum survey with certain total bandwidth and total integration time it is best to retain a high number of frequency channels across the band, and a high number of sub-integrations. However, as we increase the time and frequency resolution, the overall sensitivity of variance imaging will decrease because the noise level in each channel and subintegration increases. This leads to a 'sweet-spot' in channels and subintegrations. In turn this limits the volume in which pulsars can be found, because more distant pulsars have narrower scintillation bandwidths. We also show in D16 that the sensitivity of variance images will be lower than that of Stokes I images.

In this paper, we carry out pulsar population simulations and predict the number of pulsars that can be detected with variance imaging for future continuum surveys. We also investigate distributions and properties of pulsars detected in variance images and compare them with those of pulsars found through conventional searches. In Section 2, we describe the setup of pulsar population simulations, including 
pulsar distributions and Galactic electron-density models. In Section 3, we discuss the sensitivity of periodic search and variance imaging. In Section 4, we predict the number of pulsars that can be found with variance imaging for future continuum surveys. We discuss our results and conclude in Sections 5 and 6.

\section{PULSAR POPULATION SIMULATIONS}

We define millisecond pulsars (MSPs) as pulsars with spin period $P<20 \mathrm{~ms}$ and 'normal' pulsars with periods larger than this. According to previous studies (e.g., Levin et al. 2013; Lorimer et al. 2015), MSPs and normal pulsars have different spin period distributions and Galactic scale heights, and therefore we simulate the MSP and the normal pulsar populations separately.

To simulate pulsar populations, we used the PSRPOPPY software package (Bates et al. 2014) which is based on the PSRPOP package (Lorimer et al. 2006). The POPULATE module was used to simulate pulsars and pulsar parameters were drawn from specified distributions. The DosuRVEY module of PSRPOPPY was used to simulate existing pulsar surveys.

\subsection{Normal pulsars}

For the radial distribution of normal pulsars, we used the model of Yusifov \& Küçük (2004),

$$
\rho(r) \propto\left(\frac{r+R_{1}}{R_{\odot}+R_{1}}\right)^{a} \exp \left[-b \frac{r-R_{\odot}}{R_{\odot}+R_{1}}\right],
$$

where $\rho(r)$ is the surface density at galactocentric radius $r ; R_{\odot}$ is the Sun to the Galactic center distance; $R_{1}=0.55 \mathrm{kpc}, a=$ 1.64 and $b=4.01$ are model parameters. The distribution of normal pulsars in Galactic $z$ coordinates was approximated by a two-sided exponential (Lorimer et al. 2006),

$$
N(z) \propto \exp \left(\frac{-|z|}{E}\right)
$$

where $E=0.33 \mathrm{kpc}$ is the scale height. We used a log-normal distribution of pulse period (Lorimer et al. 2006),

$$
f(P) \propto \exp \left[-\frac{(\log P-I)^{2}}{2 J^{2}}\right],
$$

where $I=2.7$ is the mean and $J=-0.34$ is the standard deviation. The beaming model described in Smits et al. (2009) was used. We assumed a mean spectral index of -1.6 and a standard deviation of 0.35 (Lorimer et al. 1995). Pulsar luminosities were drawn from a log-normal distribution with a mean of $\left\langle\log _{10} L\right\rangle=-1.1$ and a standard deviation of $\sigma_{\log _{10} L}=0.9$ Faucher-Giguère \& Kaspi (2006).

We normalised the total number of potentially observable pulsars in the Galaxy to give a yield of 1038 pulsars for the Parkes Multibeam (PM) Pulsar Surveys (Manchester et al. 2001). This results in an underlying sample of 120000 Galactic normal pulsars, which is consistent with predictions from Faucher-Giguère \& Kaspi (2006).

\subsection{Millisecond pulsars}

We assumed the same spectral index and radial distribution for MSPs as those of normal pulsars. The distribution of MSPs in Galactic $z$ coordinates was approximated by a twosided exponential with a scale height of $0.5 \mathrm{kpc}$ (Levin et al. 2013). For the pulse period distribution of MSPs, we used a log-normal distribution following Lorimer et al. (2015),

$$
f(P) \propto \frac{1}{P} \exp \left[-\frac{(\ln P-E)^{2}}{2 F^{2}}\right]
$$

where $E=1.5$ and $F=0.58$. We used the luminosity distribution of MSPs obtained by Levin et al. (2013),

$$
N \propto\left(\frac{L}{\mathrm{mJy} \mathrm{kpc}^{2}}\right)^{-1.45},
$$

with luminosity $L$ cuts at $0.1 \mathrm{mJy} \mathrm{kpc}^{2}$ and $100 \mathrm{mJy} \mathrm{kpc} \mathrm{k}^{2}$. As discussed in Levin et al. (2013), this distribution is significantly steeper than that of normal pulsars and does not represent the luminosity distribution of MSPs below the luminosity value for the weakest pulsar in their sample. For the purpose of this paper, considering the limited sensitivity of variance imaging, our results will not be affected by the population of extremely weak MSPs.

We normalised the total number of potentially observable MSPs in the Galaxy to give 48 MSPs for the intermediate latitude part of the southern High Time Resolution Universe (HTRU) survey (Keith et al. 2010). This results in an underlying sample of 41000 Galactic MSPs.

\subsection{Galactic electron-density model}

Yao et al. (2017) model was used to estimate DMs for given pulsar Galactic longitudes and latitudes and their distances. The Yao et al. (2017) model also gives us pulsar scattering time-scales $\left(\tau_{\mathrm{sc}}\right)$ using the relation obtained by Krishnakumar et al. (2015) for the DM dependence of observed $\tau_{\text {sc }}$ values (in units of seconds and scaled to $1 \mathrm{GHz}$ assuming $\tau_{\mathrm{sc}} \approx v^{-4}, v$ is the frequency in units of $\left.\mathrm{GHz}\right)$ :

$$
\tau_{\mathrm{sc}}=4.1 \times 10^{-11} \mathrm{DM}^{2.2}\left(1.0+0.00194 \mathrm{DM}^{2}\right) .
$$

We estimate the scintillation bandwidth using

$$
2 \pi \delta v_{\mathrm{DISS}} \tau_{\mathrm{sc}}=C_{1},
$$

where $C_{1}=1.16$ for a uniform medium with a Kolmogorov wavenumber spectrum (e.g., Cordes \& Rickett 1998). The scintillation time-scale is estimated using Eq. 13 of Cordes \& Rickett (1998)

$$
\tau_{\text {DISS }}=A_{\mathrm{ISS}} \frac{\sqrt{D \delta v_{\mathrm{DISS}}}}{v V_{\mathrm{ISS}}},
$$

where $A_{I S S}=2.53 \times 10^{4} \mathrm{~km} \mathrm{~s}^{-1}, D$ is the pulsar distance and $V_{\text {ISS }}$ is the speed of interstellar diffraction pattern relative to the Earth in units of $\mathrm{km} \mathrm{s}^{-1}$. We rescale the scintillation bandwidth and time-scale to other observing frequencies using $\tau_{\text {DISS }} \propto v^{6 / 5}$ and $\delta v_{\text {DISS }} \propto v^{22 / 5}$ (e.g., Cordes $\&$ Rickett 1998). Since velocities of the observer and the ISM are normally negligible compared with the transverse velocity of pulsars $V_{\mathrm{p} \perp}$, we simply rescale the scintillation time-scale to different pulsar transverse velocities using $\tau_{\text {DISS }} \propto 100 / V_{\mathrm{p} \perp}$. For pulsar transverse velocities, we used an exponential distribution with a mean velocity of $\left\langle V_{\mathrm{p} \perp}\right\rangle=180 \mathrm{~km} \mathrm{~s}^{-1}$ (Faucher-Giguère \& Kaspi 2006). 

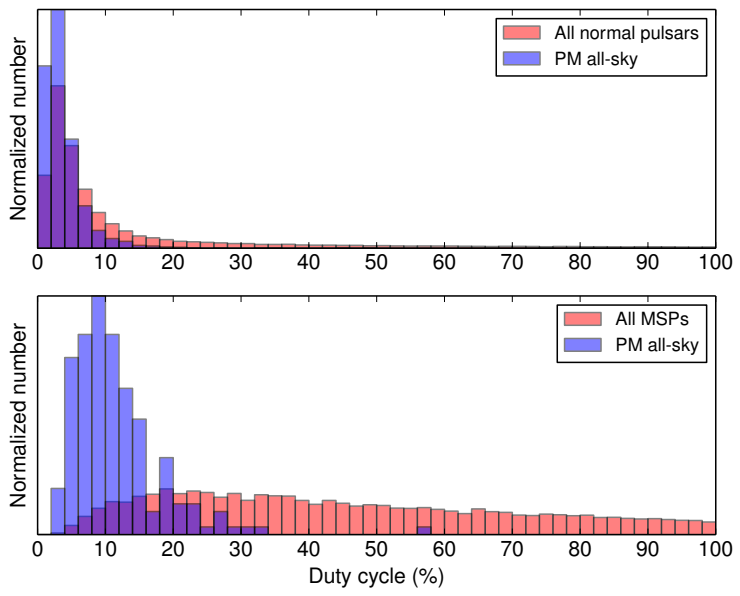

Figure 1. Histograms of duty cycles for simulated pulsars. Top panel: 120000 Galactic normal pulsars (red) and 1980 pulsars detected with the simulated PM all-sky survey. Bottom panel: 41000 MSPs (red) and 175 MSPs detected with the simulated PM all-sky surveys.

\section{SENSITIVITY OF PULSAR SEARCHES}

\subsection{Periodic search}

Conventional pulsar surveys search for strictly periodic signals. Generally, pulsars have intrinsically narrow pulses, but smearing caused by interstellar dispersion, scattering, and modulation of orbital period can all significantly increase the pulse width. The detection threshold of the apparent flux density for pulsar surveys can be calculated as

$$
S_{\min }=\frac{S / N_{\min }\left(T_{\mathrm{rec}}+T_{\mathrm{sky}}+T_{\mathrm{CMB}}\right)}{G \eta \sqrt{n_{\mathrm{pol}} t_{\mathrm{int}} \Delta v}} \sqrt{\frac{\delta}{1-\delta}} \quad \mathrm{mJy},
$$

where $S / N_{\min }$ is the threshold signal-to-noise ratio $(S / N), T_{\text {rec }}$, $T_{\text {sky }}$ and $T_{\mathrm{CMB}}$ are the receiver, sky and cosmic microwave background (CMB) temperature $(\mathrm{K}), G$ is the telescope antenna gain $(\mathrm{K} / \mathrm{Jy}), \eta$ is a survey-dependent constant $(\leq 1)$ which accounts for losses in sensitivity due to, e.g. sampling and digitisation noise, $n_{\mathrm{pol}}$ is the number of polarisation, $t_{\mathrm{int}}$ is the integration time (second) and $\Delta v$ is the observing bandwidth $(\mathrm{MHz})$. The pulse duty cycle is defined as

$$
\delta=W_{\mathrm{eff}} / P,
$$

where $W_{\text {eff }}$ is the effective pulse width and $P$ is the pulse period. The effective pulse width is given by

$$
W_{\mathrm{eff}}=\sqrt{W_{\mathrm{int}}^{2}+t_{\mathrm{samp}}^{2}+\Delta t^{2}+\tau_{\mathrm{sc}}^{2}}
$$

where $W_{\text {int }}$ is the intrinsic pulse width, $t_{\text {samp }}$ is the sampling time. $\Delta t$ is the dispersive smearing time across a frequency channel of bandwidth $\Delta v_{\mathrm{chn}}(\mathrm{MHz})$ at frequency $v(\mathrm{MHz})$ estimated as

$$
\Delta t=8.3 \times 10^{6} \mathrm{~ms} \times \mathrm{DM} \times \frac{\Delta v_{\mathrm{chn}}}{v^{3}} .
$$

The pulse smearing due to the scattering by the ionised interstellar medium, $\tau_{\mathrm{sc}}$, can be estimated using Eq. 6 .

From Eq. 9 we can clearly see that as the duty cycle increases the sensitivity of periodic search decreases, and therefore periodic searches will be biased towards low duty cycle pulsars and tend to miss pulsars smeared by dispersion, scattering and modulation of orbital period. In Fig. 1, we demonstrate this by simulating surveys and pulsar populations using distributions described in Section 2. The pulsar survey we simulated has identical parameters as the PM Pulsar Survey but with a larger sky coverage (declination $<+30^{\circ}$ ) and we call it PM all-sky survey. Fig. 1 shows that periodic searches are missing large duty-cycle pulsars and this is particularly significant for MSPs since they have much shorter spin periods than normal pulsars and are therefore heavily affected by dispersive and scattering smearing.

We note that in these simulations we did not take the smearing caused by the modulation of orbital periods of MSPs into account. For MSPs in compact binary systems, the periods will be significantly modulated if the observing time is longer than the orbital period, which will cause smearing of the pulse profile and increase the duty cycle if such modulations are not taken care of. Therefore, the sensitivity of conventional periodic searches dramatically decreases for MSPs in compact binary systems. Although various methods have been proposed to minimise the effects of orbital modulations (e.g., Ransom et al. 2003), it is still challenging to search for MSPs in binary systems.

\subsection{Variance imaging}

Statistics and simulations of detecting pulsars in variance images have been discussed in detail in D16. For a frequency resolution of $\delta v$ and a time resolution of $\delta t$, variance imaging will be most sensitive to pulsars with scintillation bandwidths of $\delta v_{\text {DISS }} \approx \delta v / 2$ and scintillation time-scales of $\delta t_{\text {DISS }} \approx \delta t / 2$. To predict the number of pulsars that can be discovered by future surveys with variance imaging, we calculate sensitivities as a function of scintillation bandwidths and time-scales for a given survey with a total bandwidth of $B$ and an integration time of $T$. For each scintillation bandwidth $\delta v_{\text {DISs }}$ and time-scale $\delta t_{\text {DISS }}$, we use a channel number of $N_{\mathrm{f}}$ and a subintegration number of $N_{\mathrm{t}}$ so that $\delta v=B / N_{\mathrm{f}}=2 \times \delta v_{\text {DISS }}$ and $\delta t=T / N_{\mathrm{t}}=2 \times \delta t_{\text {DISS }}$. This corresponds to the "matched filter" for different scintillation bandwidths and time-scales and gives us the highest sensitivity.

Under such assumptions, the number of samples in the dynamic spectrum matches the number of independent scintles. Each sample in the dynamic spectrum can then be simulated as the sum of two Gaussian random variables (for details see D16). We first determine the detection threshold in the images such that it yields a five per cent false alarm probability. Then we determine the sensitivity for which 80 per cent of the measurements exceed the detection threshold. This sensitivity is a function of the scintillation bandwidth and time-scale (see Fig. 5 in D16).

\section{DETECTING PULSARS IN VARIANCE IMAGES}

We simulate populations of normal pulsars and MSPs in the Galaxy and estimate their scintillation bandwidth and time-scale as described in Section 2. For a given continuum 
survey, the sensitivity of variance imaging as a function of scintillation bandwidth and time-scale can be estimated following Section 3.2. If the pulsar flux density is above the sensitivity corresponding to its scintillation bandwidth and time-scale, we call it as a detection, which has a five per cent false alarm probability and 80 per cent detection probability. In this way we identify all pulsars that can be detected by variance imaging with a given continuum survey.

Many of these pulsars will of course, have already been detected by conventional pulsar surveys. In order to find out the number of pulsars that only the variance imaging can find, we need to take these previous major pulsar surveys into account. We simulate pulsar surveys using the DOSURVEY module of PSRPOPPY. Pulsar surveys that cover sky areas of interest include the Parkes Southern Pulsar Survey (PSPS; Manchester et al. 1996), the Parkes Multibeam Pulsar Survey (PMPS; Manchester et al. 2001), the Swinburne Intermediate Latitude Survey (SILS; Edwards et al. 2001), the High Time Resolution Universe (HTRU; Keith et al. 2010), the pulsar survey with the Arecibo Lband Feed Array (PALFA; Cordes et al. 2006), the Arecibo all-sky $327 \mathrm{MHz}$ drift pulsar survey (AO327; Deneva et al. 2013) and the Green Band telescope $350 \mathrm{MHz}$ drift-scan survey (GBT350; Boyles et al. 2013). These simulated surveys are applied to the population of pulsars already 'discovered' in variance images thus providing the number of pulsars detected only by the variance imaging.

We carry out above simulations 100 times for normal pulsars and MSPs separately. This allows us to study DM, flux, duty cycle and sky distributions of new pulsars. Two future continuum surveys will be considered below, the EMU survey of ASKAP and an all-sky continuum survey of SKAMID.

\subsection{The EMU survey on ASKAP}

The EMU survey of ASKAP will make a deep (rms $10 \mu \mathrm{Jy} /$ beam) radio continuum survey of the entire Southern sky at $1.3 \mathrm{GHz}$, covering the entire sky south of $+30^{\circ}$ declination, with a resolution of $10 \operatorname{arcsec}$ (Norris et al. 2011). The EMU survey has a centre frequency of $1.3 \mathrm{GHz}$, a total bandwidth of $B=300 \mathrm{MHz}$, and an integration time of $T=12 \mathrm{~h}$ for each pointing (Norris et al. 2011). The default time and frequency resolutions are $10 \mathrm{~s}$ and $1 \mathrm{MHz}$ respectively, which will enable us to be sensitive to pulsars with shortest scintillation time-scales of $5 \mathrm{~s}$ and smallest scintillation bandwidths of $0.5 \mathrm{MHz}$. In order to investigate the ability of detecting new pulsars with different frequency resolutions, we also consider a frequency resolution of $0.2 \mathrm{MHz}$, a factor of five higher than the default.

For the default EMU frequency and time resolutions of $1 \mathrm{MHz}$ and $10 \mathrm{~s}$, our simulations show that the variance imaging can detect 390 normal pulsars out of the 120,000 detectable normal pulsars and 167 MSPs out of the 41000 MSPs, of which 31 normal pulsars and 38 MSPs are new. If we increase the frequency resolution of EMU to $0.2 \mathrm{MHz}$, the number of detection increases to 500 and 178 for normal pulsars and MSPs, respectively. However, increasing the frequency resolution does not increase the number of new pulsars that can be found. This is because of the limited sensitivity of EMU and the fact that the sensitivity of variance imaging decreases as we increase the frequency resolution and becomes lower than those of periodic searches.

In Fig. 2, we show the sky distribution of new pulsars (including both normal pulsars and MSPs) detected in our 100 simulations. The sky is gridded into $5.5^{\circ} \times 5.5^{\circ}$ size pointings, which roughly represents the beam size of ASKAP. The grey scale and contour show the number of pulsars detected in each pointing. We also show the sky coverage of several previous and on-going pulsar surveys with filled regions. Variance imaging of EMU will not be able to find new pulsars in regions that have been searched by deep pulsar surveys, e.g., regions searched by the PMPS, PALFA, GBT350 and AO327. At relatively high Galactic latitudes where only shallow surveys have been done EMU can find new pulsars. At Galactic longitude of $\sim 120^{\circ}$, significantly more new pulsars can be detected by variance images. This is because of the much lower electron density towards that direction where we look into the space between two spiral arms, and also because this region has not been searched by deep surveys. On the other hand, at Galactic longitude of $\sim 90^{\circ}$ where we look in the Carina-Sagittarius arm no new pulsars are found because of the dense interstellar medium.

As shown in Fig. 2, most new pulsars that can be detected by the variance imaging with EMU will be at relatively high Galactic latitudes. In Fig. 3, we show distributions of DM, flux density and duty cycle of new normal pulsars and MSPs detected by EMU (red), and compare them with those of pulsars found by the HTRU high Galactic latitude survey (blue). Variance imaging of EMU will only be able to find new pulsars with DM between $\sim 20$ and $50 \mathrm{~cm}^{-3}$ pc because the scintillation of low DM pulsars will be too weak to detect and the scintillation of high DM pulsars will be too strong for EMU to resolve the flux variance. However, we can see that variance images of EMU will be more sensitive than the HTRU high Galactic survey, and will be able to detect new faint pulsars with large duty cycles.

\subsection{Continuum surveys with the SKA}

A raft of different continuum surveys has been proposed for both SKA-MID and SKA-LOW (e.g., Prandoni \& Seymour 2015). For surveys with SKA-LOW (from $50 \mathrm{MHz}$ up to $350 \mathrm{MHz}$ ), even the most nearby pulsars will be in the strong scintillation regime (e.g., Bell et al. 2016). Variance imaging will then require very high time and frequency resolutions with a subsequent reduction in sensitivity. This implies that SKA-LOW continuum surveys will not yield many pulsars via variance imaging and we do not consider them here. Rather, we focus on continuum surveys with SKA-MID in the $1.4 \mathrm{GHz}$ band. We note, however, the SKA-LOW is a critical component for the conventional pulsar survey expected to uncover many thousands for pulsars.

An all-sky continuum survey at $1.4 \mathrm{GHz}$ with a sensitivity of $\sim 4 \mu \mathrm{Jy}$ has been proposed for SKA-MID with an observing bandwidth of $810 \mathrm{MHz}$, and an integration time is $\sim 360 \mathrm{~s}$ (Prandoni \& Seymour 2015). We assume a frequency resolution of $0.05 \mathrm{MHz}$ and a time resolution of $1 \mathrm{~s}$. Following Section 3.2 we calculate the sensitivity of variance imaging constructed with such a continuum survey as a function of scintillation bandwidth and time-scale. The observing bandwidth of $810 \mathrm{MHz}$ and frequency resolution of $0.05 \mathrm{MHz}$ allow us to detect pulsars with scintillation band- 


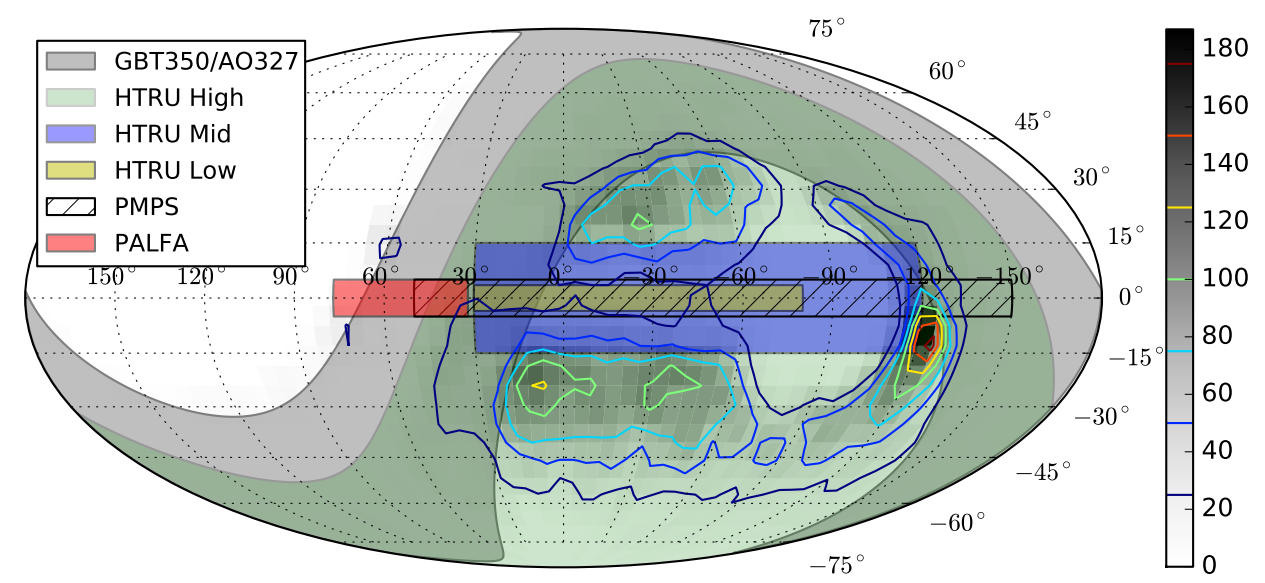

Figure 2. Sky distribution of new pulsars that can be found using variance images with EMU. The grey scale and contours show the number of new pulsars detected in 100 simulations.
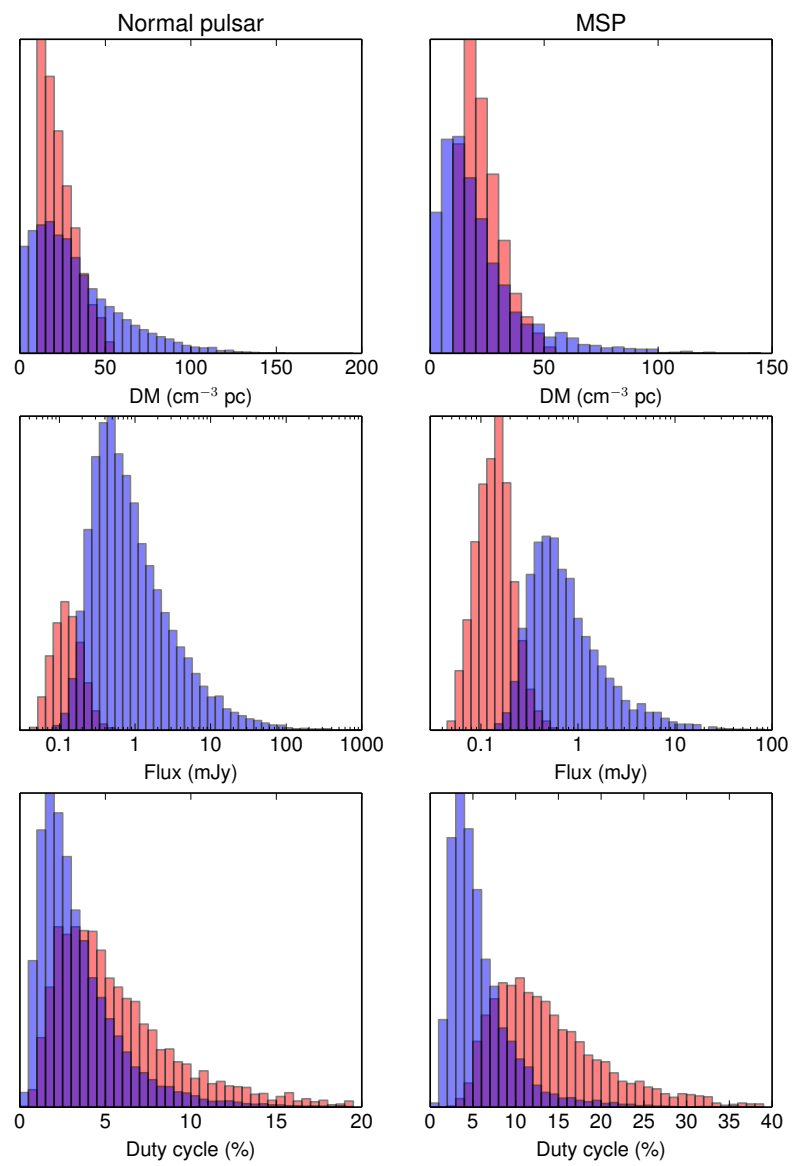

Figure 3. Distributions of new normal pulsars and MSPs detected with the EMU survey (red), compared with pulsars discovered by periodic searches (blue). For normal pulsars, distributions of DM and duty cycle are normalised so that the area underneath is unity. Other distributions are not normalised in order to show the difference. widths between $\sim 0.025 \mathrm{MHz}$ and $\sim 400 \mathrm{MHz}$. The integration time of $300 \mathrm{~s}$ and time resolution of $1 \mathrm{~s}$ enable us to be sensitive to scintillation time-scale between 0.5 and $150 \mathrm{~s}$. We also note that this imaging survey allows to use all the SKA-MID antennas and map the full field-of-view. This is not the case with the conventional pulsar search which uses only the 'core' antennas and has a limited field-of-view (e.g., Keane et al. 2015).

We separately simulate 100 populations of normal pulsars and MSPs and predict the number of new pulsars that can be detected in variance images. We find that an SKAMID all-sky continuum survey can detect 671 normal pulsars and 207 MSPs in variance images of which 143 normal pulsars and 113 MSPs are new. In Fig. 4, we show the sky distribution of new pulsars (including both normal pulsars and MSPs). In Fig. 5, we show distributions of DM, flux density and duty cycle of new normal pulsars and MSPs detected by SKA-MID (red), and compare them with those of pulsars found by the HTRU high Galactic latitude survey (blue). Compared with Fig. 2 and 3, we can see that variance imaging with SKA-mid will be able to detect new pulsars with higher DM (up to $\sim 110 \mathrm{~cm}^{-3} \mathrm{pc}$ ) because of the higher sensitivity and frequency resolution. On the other hand, variance imaging of SKA-MID will not be good at detecting low DM pulsars mostly because of the very short integration time. Again, variance imaging with SKA-MID can find pulsars with large duty cycles, especially for MSPs. The sky distribution of new pulsar is different from that of EMU as we can detect pulsars with higher DM and more pulsars can be found closer to the Galactic plane.

\section{DISCUSSION}

\subsection{Limitations of the simulation}

Critical to our simulation is the computation of scintillation bandwidth as a function of distance and galactic coordinates. The electron-density model from Yao et al. (2017) was used to predict the DM and we used a relation obtained by Krishnakumar et al. (2015) for the DM dependence of observed $\tau_{\mathrm{sc}}$ values.

Different electron-density models and different ways of 


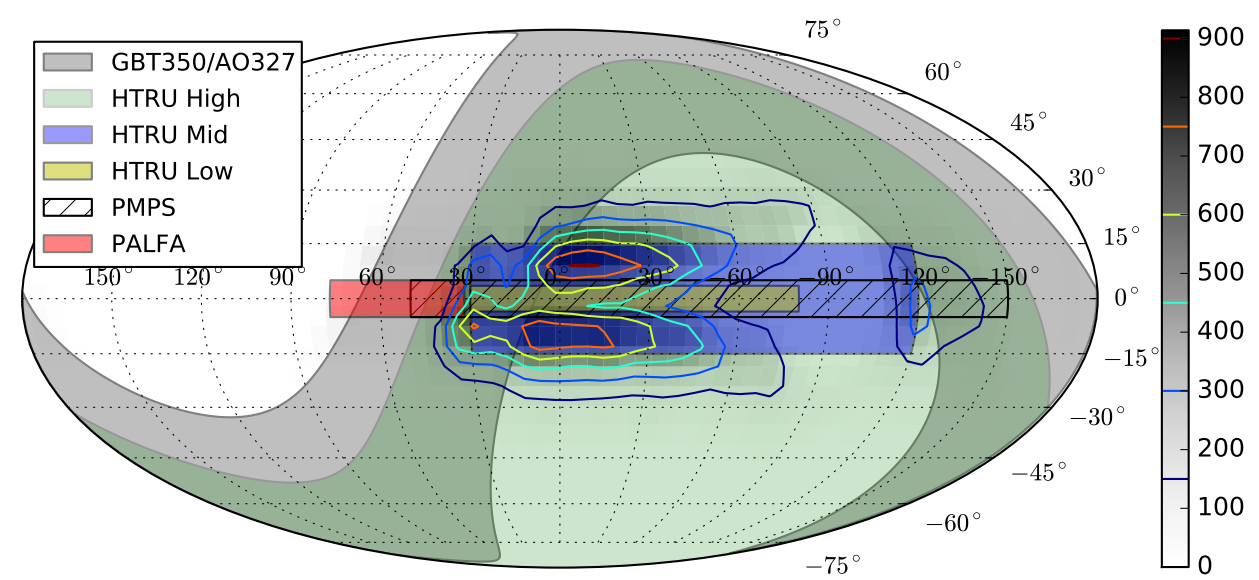

Figure 4. Sky distribution of new pulsars that can be found using variance imaging with SKA-MID. The grey scale and contours show the number of new pulsars detected in 100 simulations.
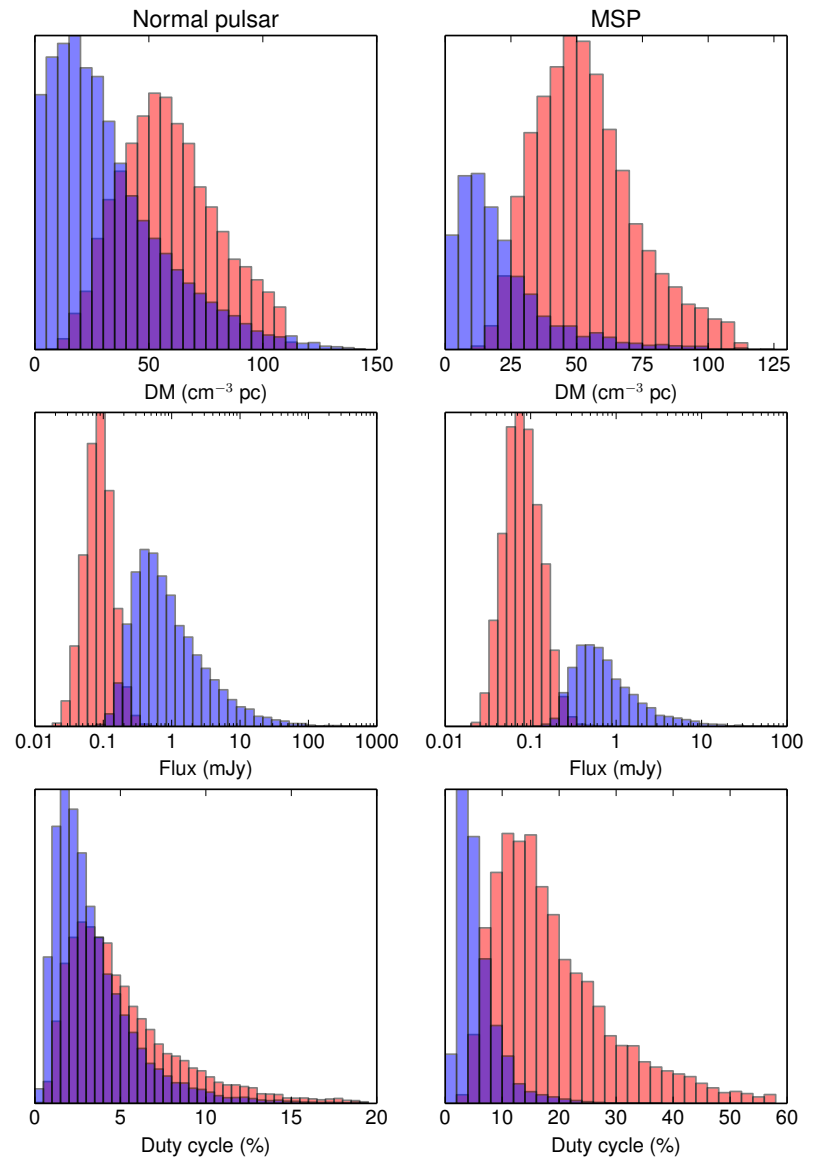

Figure 5. Distributions of new normal pulsars and MSPs detected with the SKA-MID survey (red), compared with pulsars discovered by periodic searches (blue). Distributions are not normalized.

estimating $\tau_{\mathrm{sc}}$ can result in significantly different scintillation bandwidth and time-scales for the diffractive scintillation. The widely used NE2001 electron-density model (Cordes \& Lazio 2002) predicts smaller $\tau_{\mathrm{sc}}$ and weaker scintillation (wider scintillation bandwidth) compared with Yao et al. (2017), and therefore allows the variance imaging technique to detect higher DM pulsars. Using the NE2001 model and leaving all other parameters fixed we find that EMU can detect $\sim 90$ new normal pulsars and $\sim 60$ new MSPs; the allsky continuum survey of SKA-MID can detect $\sim 230$ new normal pulsars and $\sim 150$ new MSPs.

The population of potentially observable pulsars in the Galaxy is essential for predictions of detections for future continuum surveys. Recently, Johnston \& Karastergiou (2017) show that the decay of the inclination angle $(\dot{\alpha})$ between the magnetic and rotation axes plays a critical role in pulsar evolutions and predict a total Galactic population of 20000 normal pulsars beaming towards Earth. In our simulations, we used a Galactic population of 120,000 observable normal pulsars predicted by Faucher-Giguère \& Kaspi (2006), which is much larger than the prediction of Johnston \& Karastergiou (2017). However, both Faucher-Giguère \& Kaspi (2006) and Johnston \& Karastergiou (2017) produce similar results at the flux densities under consideration here with their main differences arising at the low flux densities available only to the conventional pulsar searches with the SKA. Johnston \& Karastergiou (2017), do however predict more pulsars with large duty cycles and, potentially, variance imaging could verify this prediction.

Most MSPs are in binary systems, and some of these systems can be very compact which allow us to test gravity theories in strong gravitational field. For MSPs in relativistic binary systems, the spin period is strongly modulated by the orbital period and these systems will be very difficult to detect through periodic searches. In our simulations, we did not include orbital period of pulsars and assumed all MSPs to be isolated. However, we clearly show that variance images detect more large duty-cycle MSPs, and therefore should be more sensitive to MSPs in relativistic binary systems than periodic searches.

\subsection{Implications}

Our simulations show that variance imaging of the EMU survey of ASKAP should find 30 new normal pulsars and $\sim 40$ new MSPs, and an all-sky continuum survey with SKA- 
MID should detect $\sim 140$ new normal pulsars and $\sim 110$ new MSPs. Our results indicate that

- at $1.4 \mathrm{GHz}$, variance imaging with future surveys will only be able to detect pulsars with DM below $\sim 120 \mathrm{~cm}^{-3} \mathrm{pc}$ and hence are most likely to find pulsars at relatively high Galactic latitudes. To detect high DM pulsars requires higher frequency surveys with high sensitivity.

- variance imaging with EMU has only limited sensitivity and is unlikely to find a large number of new pulsars. However, it is more sensitive than current pulsar surveys at high Galactic latitudes in the southern hemisphere and so variance imaging with EMU provides us an efficient way to search for pulsars at these high latitudes.

- when compared with conventional pulsar searches, variance imaging is better at identifying pulsars with large duty cycles, and therefore is a powerful tool for finding MSPs, whose duty cycles are normally larger than normal pulsars. The spin-periods of MSPs in relativistic binary systems will be heavily modulated by their orbital velocity, which results in large duty cycles if the orbital motion is not corrected. Variance imaging can help us identify such systems.

- variance imaging with SKA-MID continuum surveys provides complementarity to the conventional pulsar searches planned for SKA-MID and SKA-LOW. Keane et al. (2015) predict that an all-sky pulsar survey with SKA-LOW can detect $\sim 900 \mathrm{MSPs}$, and the DM distribution of these MSPs peaks at $\sim 160 \mathrm{~cm}^{-3} \mathrm{pc}$. Our simulations show that variance imaging with SKA-MID all-sky continuum survey can detect $\sim 210$ MSPs with DM below $\sim 120 \mathrm{~cm}^{-3}$ pc.

- variance imaging can help us distinguish pulsars from other compact radio sources, but is less sensitive than continuum imaging and is limited to relatively low DM pulsars. Variance imaging is only one possible avenue for finding pulsars in continuum images; searches in polarisation, rotation measure and circular polarisation are other options.

\subsection{Implementation}

The next stage for this project is to implement these ideas on existing data and to plan for large-scale reduction of the future ASKAP surveys. Much work is still needed to deal with the practicalities of real data. Radio frequency interference needs to be mitigated. It remains unclear whether or not deconvolution of the images will be necessary and this will likely depend on snapshot $\mathrm{u}-\mathrm{v}$ coverage. A technique for filtering out sources with smoothly varying spectra (e.g., power-law) needs to be developed. In general, however, we expect the generation of variance images to be computationally cheap compared to the cost of generating the continuum images.

We also need to consider the false alarm threshold. In this paper this is set, somewhat arbitrarily, to five per cent. Raising this level will result in the detection of more pulsars but will also increase the number of potential candidates needing follow-up observations. In order to avoid this number becoming too large, other criteria can be used. For example, if a radio source is identified with an optical galaxy or if it is extended it can be ruled out as being a pulsar even if it shows up in the variance image. The false alarm threshold will therefore be survey specific and will depend on a raft of different inputs.
Finally, we note that continuum images with existing telescopes can already be used to detect pulsars (e.g., Kaplan et al. 1998, 2000; Han \& Tian 1999; Crawford et al. 2000; Kouwenhoven 2000). Bell et al. (2016) studied the variability of pulsars in the southern sky using $154 \mathrm{MHz}$ Murchison Widefield Array imaging observations. While the limited sensitivity of current all-sky continuum surveys makes it difficult to discover new pulsars, ultra-deep images have been used to search for pulsars in the Galactic center (e.g., Lazio \& Cordes 2008; Bhakta et al. 2017). In order to detect pulsars in variance images, we need to have enough bandwidth, frequency and time resolution to resolve diffractive scintillation, and have good snapshot sensitivity and $\mathrm{u}-\mathrm{v}$ coverage. This can already be achieved by recent continuum surveys (Dai et al. 2016), and can be combined with conventional pulsar searches to identify extreme pulsars.

\section{CONCLUSIONS}

The optimum method for finding pulsars is through timeseries analysis which involves de-dispersion, Fourier transforms and harmonic summing. These techniques have reaped 2500 pulsars to date, with the SKA expected to find in excess of 10000 pulsars. Interferometric pulsar searches come with a heavy computational cost, and it is currently envisaged that the SKA survey can only make use of limited number of antennas and a limited field of view.

Other, complementary techniques for uncovering pulsars in continuum surveys, which essentially come 'for free' along with the imaging, are therefore worth exploring. Such techniques include examining steep spectrum objects, searching in Rotation Measure space, searching in circular polarisation and through the scintillation properties of pulsars. We have examined the viability of finding pulsars via variance imaging and show that while the yield of pulsars from the EMU survey on ASKAP is small, surveys with SKA-MID can significantly add to the haul of MSPs in particular.

Although variance imaging with EMU and SKA-MID is only sensitive to pulsars with relatively low DM (less than $\sim 120 \mathrm{~cm}^{-3} \mathrm{pc}$ ), they are equally sensitive to pulsars with different duty cycles, and are therefore better at identifying pulsars with large duty cycles than conventional pulsar searches. Variance imaging is likely to be a powerful technique for finding pulsars in relativistic binary systems.

\section{REFERENCES}

Bates S. D., Lorimer D. R., Rane A., Swiggum J., 2014, MNRAS, 439,2893

Bell M. E., et al., 2016, ArXiv:1605.09100,

Bhakta D., Deneva J., Frail D. A., de Gasperin F., Intema H. T., Jagannathan P., Mooley K. P., 2017, preprint, (arXiv: 1703.04804)

Boyles J., et al., 2013, ApJ, 763, 80

Cordes J. M., Lazio T. J. W., 2002, ArXiv Astrophysics e-prints astro-ph/0207156,

Cordes J. M., Rickett B. J., 1998, ApJ, 507, 846

Cordes J. M., et al., 2006, ApJ, 637, 446

Crawford F., Kaspi V. M., Bell J. F., 2000, AJ, 119, 2376

Dai S., Johnston S., Bell M. E., Coles W. A., Hobbs G., Ekers R. D., Lenc E., 2016, MNRAS, 462, 3115 
Deneva J. S., Stovall K., McLaughlin M. A., Bates S. D., Freire

P. C. C., Martinez J. G., Jenet F., Bagchi M., 2013, ApJ, 775, 51

Edwards R. T., Bailes M., van Straten W., Britton M. C., 2001, MNRAS, 326, 358

Faucher-Giguère C.-A., Kaspi V. M., 2006, ApJ, 643, 332

Han J. L., Tian W. W., 1999, A\&AS, 136, 571

Johnston S., Karastergiou A., 2017, MNRAS, 467, 3493

Kaplan D. L., Condon J. J., Arzoumanian Z., Cordes J. M., 1998, ApJS, 119, 75

Kaplan D. L., Cordes J. M., Condon J. J., Djorgovski S. G., 2000, ApJ, 529, 859

Keane E., et al., 2015, Advancing Astrophysics with the Square Kilometre Array (AASKA14), p. 40

Keith M. J., et al., 2010, MNRAS, 409, 619

Kouwenhoven M. L. A., 2000, A\&AS, 145, 243

Krishnakumar M. A., Mitra D., Naidu A., Joshi B. C., Manoharan P. K., 2015, ApJ, 804, 23

Lazio T. J. W., Cordes J. M., 2008, ApJS, 174, 481

Levin L., et al., 2013, MNRAS, 434, 1387

Lorimer D. R., Yates J. A., Lyne A. G., Gould D. M., 1995, MNRAS, 273, 411

Lorimer D. R., et al., 2006, MNRAS, 372, 777

Lorimer D. R., et al., 2015, MNRAS, 450, 2185

Manchester R. N., et al., 1996, MNRAS, 279, 1235

Manchester R. N., et al., 2001, MNRAS, 328, 17

Norris R. P., et al., 2011, Publ. Astron. Soc. Australia, 28, 215

Prandoni I., Seymour N., 2015, Advancing Astrophysics with the Square Kilometre Array (AASKA14), p. 67

Ransom S. M., Cordes J. M., Eikenberry S. S., 2003, ApJ, 589, 911

Smits R., Lorimer D. R., Kramer M., Manchester R., Stappers B., Jin C. J., Nan R. D., Li D., 2009, A\&A, 505, 919

Yao J. M., Manchester R. N., Wang N., 2017, ApJ, 835, 29

Yusifov I., Küçük I., 2004, A\&A, 422, 545

This paper has been typeset from a $\mathrm{TE}_{\mathrm{E}} \mathrm{X} / \mathrm{L} \mathrm{A} \mathrm{T} \mathrm{X}$ file prepared by the author. 